$5-1-2010$

\title{
A Realistic Mobility Model for Wireless Networks of Scale-Free Node Connectivity
}

Sunho Lim

Texas Tech University, sunho.lim@ttu.edu

Chansu Yu

Cleveland State University, c.yu91@csuohio.edu

Chita R. Das

The Pennsylvania State University, das@cse.psu.edu

Follow this and additional works at: https://engagedscholarship.csuohio.edu/enece_facpub

Part of the Electrical and Computer Engineering Commons

How does access to this work benefit you? Let us know!

\section{Repository Citation}

Lim, Sunho; Yu, Chansu; and Das, Chita R., "A Realistic Mobility Model for Wireless Networks of Scale-Free Node Connectivity" (2010). Electrical Engineering \& Computer Science Faculty Publications. 239.

https://engagedscholarship.csuohio.edu/enece_facpub/239

This Article is brought to you for free and open access by the Electrical Engineering \& Computer Science Department at EngagedScholarship@CSU. It has been accepted for inclusion in Electrical Engineering \& Computer Science Faculty Publications by an authorized administrator of EngagedScholarship@CSU. For more information, please contact library.es@csuohio.edu. 


\title{
A realistic mobility model for wireless networks of scale-free node connectivity
}

\author{
Sunho Lim*
}

Department of Computer Science, Texas Tech University, Lubbock, TX 79409, USA

E-mail: sunho.lim@ttu.edu

${ }^{*}$ Corresponding author

\section{Chansu Yu}

Department of Electrical and Computer Engineering, Cleveland State University, Cleveland, $\mathrm{OH} 44115$, USA

E-mail: c.yu91@csuohio.edu

\section{Chita R. Das}

Department of Computer Science and Engineering, The Pennsylvania State University, University Park, PA 16802, USA

E-mail: das@cse.psu.edu

\begin{abstract}
Recent studies discovered that many of social, natural and biological networks are characterised by scale-free power-law connectivity distribution. We envision that wireless networks are directly deployed over such real-world networks to facilitate communication among participating entities. This paper proposes Clustered Mobility Model (CMM), in which nodes do not move randomly but are attracted more to more populated areas. Unlike most of prior mobility models, CMM is shown to exhibit scale-free connectivity distribution. Extensive simulation study has been conducted to highlight the difference between Random WayPoint (RWP) and CMM by measuring network capacities at the physical, link and network layers.
\end{abstract}

Keywords: connectivity distribution; mobile communication; mobility model; network capacity; random waypoint mobility; scale-free wireless networks.

Reference to this paper should be made as follows: Lim, S., Yu, C. and Das C.R. (2010) 'A realistic mobility model for wireless networks of scale-free node connectivity', Int. J. Mobile Communications, Vol. 8, No. 3, pp.351-369.

Biographical notes: Sunho Lim received the BS Degree (summa cum laude) in Computer Science and the MS Degree in Computer Engineering, Hankuk Aviation University, Korea, in 1996 and 1998, respectively. 
He received the $\mathrm{PhD}$ Degree in Computer Science and Engineering from the Pennsylvania State University, University Park, in 2005. He is currently an Assistant Professor in the Department of Computer Science, Texas Tech University. His research interests are in the areas of wireless mobile P2P networks, mobile data management, and network security. $\mathrm{He}$ is a member of the IEEE.

Chansu $\mathrm{Yu}$ received BS and MS in Electrical Engineering from the Seoul National University, Korea, in 1982 and 1984, respectively, and $\mathrm{PhD}$ in Computer Engineering from the Pennsylvania State University in 1994. $\mathrm{He}$ is currently an Associate Professor in the Department of Electrical and Computer Engineering at the Cleveland State University in Cleveland, Ohio. He has authored/co-authored more than 60 technical papers and numerous book chapters in the areas of mobile networking, performance evaluation and parallel and distributed computing. He is a member of the IEEE and IEEE Computer Society.

Chita R. Das received the MSc Degree in Electrical Engineering from the Regional Engineering College, Rourkela, India, in 1981, and the $\mathrm{PhD}$ Degree in Computer Science from the Center for Advanced Computer Studies, University of Louisiana, Lafayette, in 1986. Since 1986, he has been with the Pennsylvania State University, where he is currently a professor in the Department of Computer Science and Engineering. His main areas of interest are parallel and distributed computer architectures, cluster computing, mobile computing, Internet QoS, multimedia systems, performance evaluation, and fault-tolerant computing. He has served on the editorial boards of the IEEE Transactions on Computers and the IEEE Transactions on Parallel and Distributed Systems. He is a fellow of the IEEE and a member of the ACM.

\section{Introduction}

Since node mobility greatly affects the performance of mobile wireless networks, a realistic mobility model is critically required to study such networks. Random WayPoint (RWP) mobility model (Johnson and Maltz, 1996) is a synthetic mobility model, which is extensively used in studying Mobile Ad hoc Networks (MANETs). Recently, numerous efforts have been made to obtain statistics from either synthetic (e.g., RWP) or real-life traces. For example, (Bettstetter et al., 2004; Yoon et al., 2003; Jardosh et al., 2003) analysed spatial and temporal characteristics of RWP and (Hsu et al., 2007; Rhee et al., 2007) attempted to characterise mobility pattern based on real-life mobility data. However, most of previous works on mobility models do not consider any explicit rationale behind the mobility behaviour. In other words, a node does not make a move without referring to a certain demand for the move (Oppenheim, 1995; Centonza et al., 2006). In the context of RWP, the selection of a waypoint is random within a given terrain area and does not take a certain behavioural rule or rationale into consideration. 
On the contrary, this paper proposes a behaviour-based mobility model, called Clustered Mobility Model (CMM), in which nodes move for a reason. They tend to move towards a certain waypoint where more nodes are already present. Mobility rationale incorporated in CMM is neither comprehensive nor universal. In fact, a totally opposite rationale can also be employed in a different scenario. For example, a node tries to move to a less populated area to avoid congestion. However, our intention in this paper is to suggest that a mobility model can better represent real-life scenarios when the rationale behind the move is captured and incorporated in the mobility model. It is not difficult to expect that CMM results in heterogeneous node densities across the terrain area. Some subareas are highly populated with nodes but others are sparse. It could also lead to the creation of highly connected nodes, called hubs, as well as clustering or gathering of nodes around the hubs. This is a clear contrast to the conventional random mobility models such as RWP (Johnson and Maltz, 1996), in which nodes are scattered almost uniformly in the network.

Contributions of this paper are three-fold: First, the paper proposes a realistic mobility model where nodes move for a reason. We believe this study opens up future development of mobility models based on more sophisticated node behaviour. Second, although hub nodes and their adverse impact on network performance have been addressed recently (Kawadia and Kumar, 2003; Wang and Li, 2002a), none of these research pays attention to how hub nodes are created and how to model them. The proposed CMM explains one possible behavioural scenario. Third, to demonstrate how CMM affects the network performance, this paper defines network capacity at physical and link layers and compares CMM and RWP in terms of the two capacities. While network capacity is a fundamental measure of a wireless network, previous studies have evaluated at one particular level, which may not accurately assess the true performance of a network or a system.

This paper is organised as follows. Section 2 summarises recent work on small-world (Strogatz, 2001) and scale-free networks (Barabasi and Bonabeau, 2003) to provide a brief introduction on hubs and node clustering. Section 3 overviews previous research on mobility models by focusing on how non-homogeneous node distribution arises and how it is modelled. Section 4 proposes CMM. Section 5 is devoted to the performance analysis of CMM and its comparison to the conventional RWP in terms of network capacities. Section 6 concludes our work.

\section{Background: scale-free networks}

Recently there has been considerable interest in the structure and dynamics of large complex networks found in natural, technological and social networks (Strogatz, 2001; Barabasi and Bonabeau, 2003). Random graphs, pioneered more than 40 years ago (Erdös and Rényi, 1960), are often used to model such complex networks. However, it has consistently been shown that topologies of real-life networks are not uncorrelated random graphs (Németh and Vatty, 2003). They include electric power grid, the World Wide Web, the internet backbone, collaboration and citation networks in the scientific community, and US airline connection networks. Strogatz (2001) introduced the concept of small-world graphs, which exhibits small-world property as well as node clustering. 
Barabasi and Bonabeau (2003) have studied the World Wide Web and found that its connectivity (node degree) distribution follows a power law. It renders highly connected nodes or hubs to have a large chance of occurring, which is unusual in random networks. They call networks containing hubs scale-free in the sense that some of the hubs have a seemingly unlimited connectivity and no node is typical of the others. Formally speaking, a network is called scale-free if the moments $\left\langle k^{\nu}\right\rangle$ for $\nu \geq \nu_{\max }$ do not exist (diverge) in the limit of $n \rightarrow \infty$, where $k$ and $n$ denote the connectivity (node degree) and the number of nodes in the network, respectively.

A scale-free network has been explained with two generic mechanisms: incremental growth and preferential attachment (Barabasi and Bonabeau, 2003). As new nodes in a scale-free network appear, they tend to connect to the more connected nodes. In other words, the probability $\varphi_{i}$ that a new node will be connected to a node $i$ depends on the connectivity $k_{i}$ of that node, i.e., $\varphi_{i}=\frac{k_{i}+1}{\Sigma_{j}\left(k_{j}+1\right)}$ (Barabasi and Bonabeau, 2003). Medina et al. built a parameterised topology generator, called BRITE, which takes incremental growth and preferential attachment into account to explore power laws in internet topologies (Medina et al., 2000). It was shown analytically that these mechanisms lead to the power-law connectivity distribution, i.e., $p_{k} \propto k^{-\beta}$, where $p_{k}$ denotes the probability that a node has $k$ connectivity (Albert and Barabasi, 2000). For reasons not yet known, the value of $\beta$ tends to fall between 2 and 3 (Barabasi and Bonabeau, 2003). An extended model has been introduced in Albert and Barabasi (2000), Chen et al. (2002) to give a more realistic description of the local processes such as rewiring. It allows for some additional flexibility in the formation of networks by removing links connected to certain nodes and replacing them by new links in a way that effectively amounts to a local type of re-shuffling connections.

It is important to note that majority of small-world and scale-free networks assume relational graph model where distance is measured only by the graph itself. MANETs as well as some real-life networks such as routers of internet and transportation networks are embedded in physical Euclidean space and possess a geography in addition to their topology. The spatial location of nodes and their geographical proximity determines the connectivity among the nodes (Herrmann et al., 2003; Németh and Vatty, 2003). In this type of spatial graphs, two neighbours of a node have a better chance to be neighbours with each other, which is not necessarily true in relational graphs. Large connectivities are usually obtained in high density regions, called hub areas, and therefore, small-world graphs exhibit scale-free property or vice versa unlike in relational graphs (Herrmann et al., 2003).

The above-mentioned studies on small-world and scale-free networks have motivated us to consider a realistic mobility model because preferential attachment could be a key mobility rationale. All the concepts and theories developed for small-world and scale-free networks can also be utilised in developing a new mobility model, which is discussed in Section 4.

\section{Related work}

Performance of MANETs is greatly affected by the mobility of nodes as routing algorithms need to discover routes in the presence of frequent link changes (Alchaita, 2008; Jolly and Latifi, 2007). Simulation tools used in ad hoc network studies try to mimic the distribution and movement pattern of nodes in 
real-life scenarios. This section discusses several mobility models proposed in the literature and explains how they distribute nodes in the terrain area.

\subsection{Mobility models}

Mobility models can be divided into individual and group mobility models, where the difference lies whether or not the position and movement pattern of a mobile node is independent of others (Camp et al., 2002). Random Walk or Brownian Motion is an individual mobility model that emulates the erratic movement of various entities in nature, and also, in wireless mobile networks (Bar-Noy et al., 1995). The main disadvantage of this model is that it results in sharp turns and sudden stops. RWP has been proposed in Johnson and Maltz (1996) and overcomes the shortcomings of Random Walk model. In RWP, each node pauses for a predefined amount time, moves toward a randomly selected waypoint with a random speed chosen uniformly from $\left[V_{\min }, V_{\max }\right]$, and repeats the process. The model has been extensively used in ad-hoc network simulations and has become a reference model for other mobility models. Mobility models such as Manhattan Mobility model and Obstacle Mobility model (Camp et al., 2002) are a step forward towards realistic mobility models, but their applicability is limited to some particular applications and the selection of a destination node and initial distribution is based on RWP.

Another interesting idea of adding practicality in mobility models is group mobility, wherein a group of nodes share a common mobility pattern. In Random Point Group Mobility (RPGM) model (Wang and Li, 2002a), each group has a logical centre and the movement pattern of a logical centre controls that of its member nodes including speed, direction, and acceleration, etc. In Reference Velocity Group Mobility (RVGM) model (Wang and Li, 2002b), each mobility group is characterised by a common mean group velocity, that acts as the reference velocity for the nodes in a group. The RWP has been extended to incorporate the concept of group in Cano et al. (2004). Nodes in the network are evenly divided into a number of groups to examine the impact of group scenarios on network performance. Virtual Track (VT) is another group mobility model, which can simulate group dynamics such as group split and merge (Zhou et al., 2004).

\subsection{Node distribution}

It is important to observe that most of the above-mentioned mobility models are still based on random mobility pattern without referring to a certain rationale behind the move and thus, produce uniformly random distribution of nodes. For example, in RWP, initial positions of nodes as well as target waypoints are randomly and independently selected. However, it is well known that mobility causes the undesirable concentration of nodes at the centre of a network as time progresses (Yoon et al., 2003; Bettstetter et al., 2003; Blough et al., 2004). This is because the shortest path between two consecutive waypoints would pass across the centre of the network area more probably than the boundaries. In this paper, a simple modification has been made to RWP such that it leads to a uniformly random node distribution. It follows the same movement as in the original RWP except that the network is wrapped around. In other words, the shortest path to a waypoint 
is determined assuming that the left and right sides of the network (and top and bottom sides) are adjacent.

Now, consider the spatial distribution of nodes in a network area with RWP. Assume that the entire area is divided into $s_{t}$ equal-sized subareas. Each node is positioned in a particular subarea $i$ with independent probability, $\varphi_{i}$, where $\varphi_{i}=\frac{1}{s_{t}}$ for all $i$ 's. The probability $p_{k}$ that a subarea has exactly $k$ nodes is given by the binomial distribution, $p_{k}=C_{k}^{n} \varphi^{k}(1-\varphi)^{n-k}$, where $n$ is the total number of nodes. As a limiting case, this probability becomes the well-known Poisson distribution $p_{k}=\frac{z^{k} e^{-z}}{k !}$, where $z$ is the mean number of nodes in a subarea, which is equal to $n \varphi$ or $\frac{n}{s_{t}}$. Both binomial and Poisson distributions are strongly peaked about the mean $z$, and decays rapidly as a function of $\frac{1}{k !}$ (Watts and Strogatz, 1998). In other words, with the uniformly random node distribution, the majority of subareas have similar number of nodes $\left(z\right.$ or $\left.\frac{n}{s_{t}}\right)$ and any significant deviation from the average case (e.g., a subarea with a large number of nodes) is extremely rare.

Figure 1 Node distribution in an example ad hoc network: (a) rescue team at ground zero (Sullivan, 2001) and (b) node density distribution (see online version for colours)

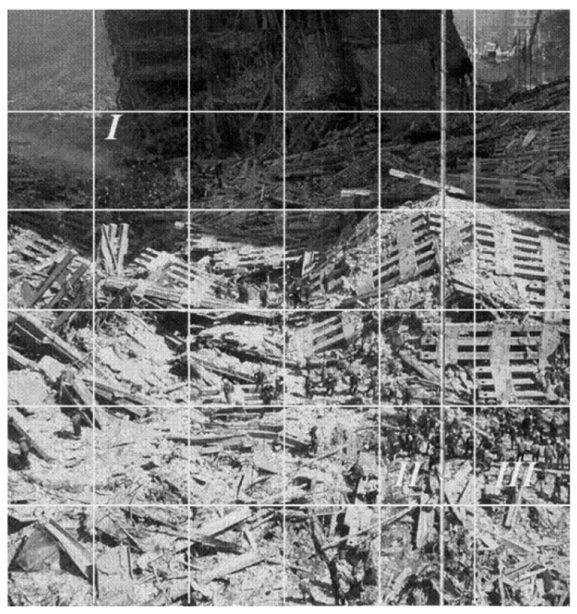

(a)

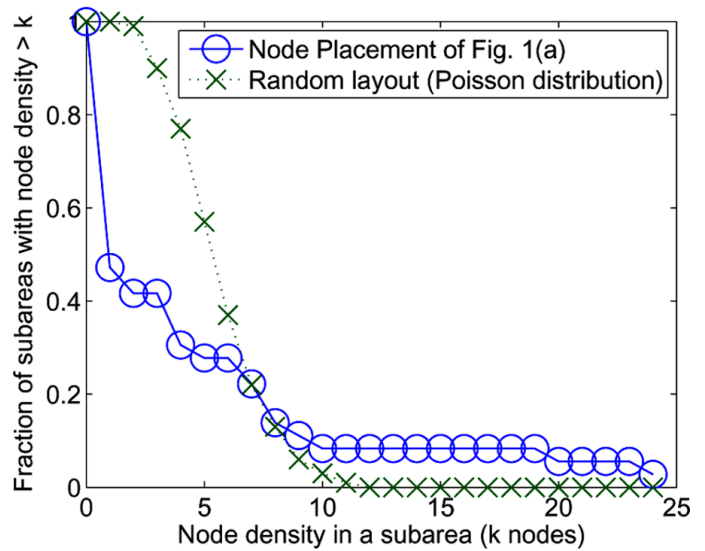

(b) 
In a real network of mobile nodes, however, the node distribution can be very different from the Poisson distribution. For example, Figure 1(a) shows an example of a disaster area, where the infrastructure-less ad hoc network is well suited for supporting communication. Many rescue team members gather at three subareas (hubs), denoted as I, II and III in the figure, which may be a base camp or have many casualties. The three subareas out of $36\left(s_{t}=36\right)$ include about the half of the total rescue team members (66 out of 137). Figure 1(b) shows the node density distribution of the disaster area in Figure 1(a) as well as that of the uniformly random node distribution that follows the Poisson distribution. It is clear from Figure 1(b) that RWP does not model the node distribution of a real ad hoc network situation. As evident in Figure 1(b), the node distribution of the Ground Zero example (Sullivan, 2001) contains a heavy-tail unlike the Poisson distribution. Heavy-tail can be modelled by a power law distribution and the main cause of this phenomenon can be explained using the principle of preferential attachment as described in Section 2. The CMM model produces the heavy-tail distribution.

\section{Clustered Mobility Model}

This section presents a behaviour-based mobility model called the Clustered Mobility Model (CMM), which produces node clustering and hubs as in the example network in Figure 1. Note that the degree of node clustering is controllable in CMM. The network evolution process is described in Sections 4.1 and 4.2 analyses the characteristics of CMM.

\subsection{Synthesis of CMM}

The CMM model consists of two steps as in RWP, the first being to generate the initial layout and the second being the selection of waypoints to induce mobility. The two steps correspond to growth (Barabasi and Bonabeau, 2003) and rewiring (Albert and Barabasi, 2000; Chen et al., 2002) (see Section 2). Pause time and node speed are selected as in RWP. The difference lies in selecting the initial layout and waypoints. The entire simulation area is logically divided into a number of subareas. During both the growth and rewiring steps, a node is attracted to a subarea $i$ with a higher probability than a subarea $j$ if $i$ has more number of nodes than $j$. Note that in Section 2, $i, k_{i}$ and $\varphi_{i}$ denote a node, degree of node $i$ and the probability that a new node connects to node $i$, respectively. Here, they denote a subarea, the number of nodes in subarea $i$, and the probability that a new node is positioned in subarea $i$.

The first step of CMM (growth) is to generate an initial layout. Initially all subareas have no nodes, and therefore, the probability of a subarea being assigned the next node is equal. But as a new node is assigned to a subarea, its probability increases or decreases depending on the present number of nodes in that subarea. For example, if a subarea $i$ has $k_{i}$ nodes, its probability, $\varphi_{i}$, is $\frac{\left(k_{i}+1\right)^{\alpha}}{\Sigma_{j}\left(k_{j}+1\right)^{\alpha}}$, where $\alpha$ is the clustering exponent. During the process, some subareas will have a higher probability than others and will become the hub areas. Within the chosen subarea, a node is randomly located. The growth process ends when all the pre-determined number of nodes have been assigned subareas and are positioned in the subareas. Final $\varphi_{i}$ 's will be used in the next step and would not be changed during the simulation. 
The second step of CMM (rewiring) is to induce mobility. Each node is rewired from one subarea to another when it repeats the pause-and-mobility cycle. A waypoint is selected by, first, choosing a subarea and then choosing a position within that subarea. The choice of a subarea is again based on the principle of preferential attachment using $\varphi_{i}$ 's. Position inside the chosen subarea is randomly chosen. The node selects a speed, which is uniformly distributed between $\left[V_{\min }, V_{\max }\right]$ as in RWP. Here, $V_{\min }$ is set to nonzero so that the average node speed does not diminish as time progresses (Bettstetter et al., 2004; Yoon et al., 2003). The overall CMM algorithm is summarised in Figure 2.

Figure 2 The pseudo code of the CMM

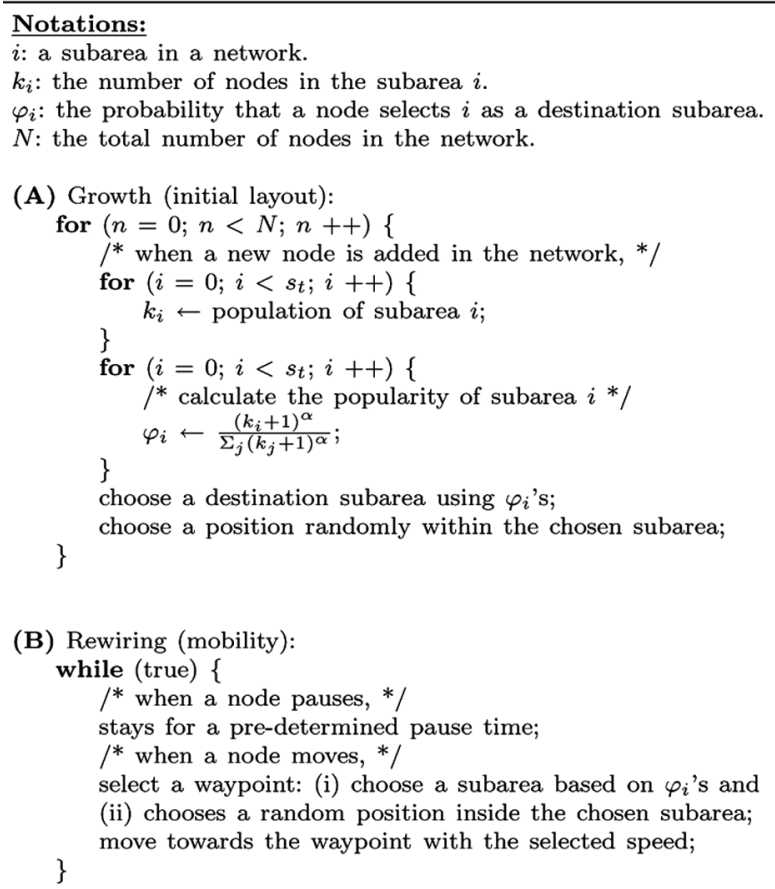

There are two additional issues to be noted. First, two different interpretations of the preferential attachment mechanism are possible; attracted to a node or to a subarea. However, they are in fact not very different in spatial graphs as explained in Section 2. A subarea with a hub node is essentially a hub subarea because a large number of connectivity of a hub node directly translates to a large number of nodes in the proximity. Second, the size of a subarea is carefully chosen so that a node in a subarea directly connects to most of the other nodes in the same subarea but those in neighbouring subareas may or may not connect depending on their locations in the corresponding subareas. This paper uses the transmit range of the radio device (i.e., $250 \mathrm{~m}$ ) as each side of a subarea.

\subsection{Analysis of CMM}

This subsection shows an example initial node distribution produced by the growth process of CMM and the node distribution at steady state when the simulation 
time progresses enough. It is obvious that they are different as the degree of node clustering is greatly reduced during the mobility (rewiring) step. This is because nodes are not rewired to the chosen waypoints immediately. Rather, they need to travel in between the waypoints. While waypoints are chosen based on the preferential attachment mechanism, the position of nodes between the waypoints is considered random. In order to quantify this effect, we define a mobility fraction, $\xi$, as the fraction of time a node moves during its lifetime. Higher the node speed, lower is the mobility fraction, and, smaller the pause time, lower is the mobility fraction. Note that network size also affects the mobility fraction because a node moves for a longer duration to reach a destination in a larger network.

Consider an example network where a node chooses its speed from $[5,20] \mathrm{m} / \mathrm{s}$ and pauses for $60 \mathrm{~s}$ in a $3000 \times 3000 \mathrm{~m}^{2}$ network area. It is not difficult to show that the average distance between two waypoints is about $1148 \mathrm{~m}$ and that the average move time is about $106 \mathrm{~s}$. Since pause time is $60 \mathrm{~s}, \xi=\frac{106}{60+106}=64 \%$. It can be interpreted that a node is uniformly randomly located in the network for $64 \%(\xi)$ of the time but located in a scale-free fashion for the rest of the time $(36 \%$ or $1-\xi)$.

Figure 3 compares node distributions of CMM $(\alpha=1.2$ and $\alpha=1.4)$ with RWP. The total number of nodes is assumed to be 1000 in the network area of $3000 \times 3000 \mathrm{~m}^{2}$, and a node speed is chosen from [5, 20] m/s. From Figure 3(a) and (d), it would be safe to say that RWP provides uniformly random as well as consistent node distribution during the simulation. In CMM(1.2), the level of node concentration is higher in Figure 3(b) than in Figure 3(e), which are at initial and steady state stages. In Figure 3(c) and (f), the same phenomenon is observed, while nodes are more cluttered than the case with $\alpha=1.2$.

Figure 3 Node distributions in RWP and CMM models $(N=1,000,5 \leq v \leq 20.0(\mathrm{~m} / \mathrm{s})$, and $\left.T_{\text {pause }}=106(\mathrm{~s})\right) . \alpha$ is set to be $1.2(\mathrm{CMM}(1.2))$ for $(\mathrm{b})$ and (e) and 1.4 $(\mathrm{CMM}(1.4))$ for (c) and (f). Subfigures (a), (b), and (c) show the initial layouts Subfigures (d), (e), and (f) show the steady-state layouts (see online version for colours)

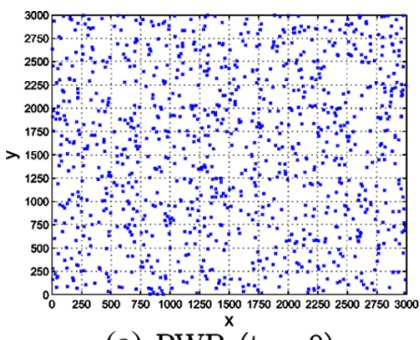

(a) $\operatorname{RWP}^{x}(t=0)$

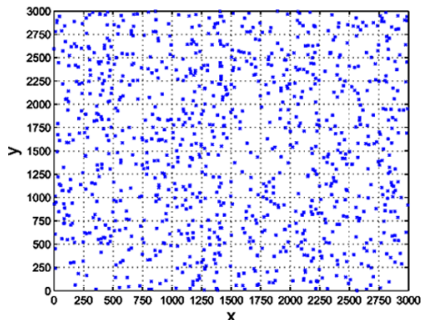

(d) RWP (t $=10,000)$

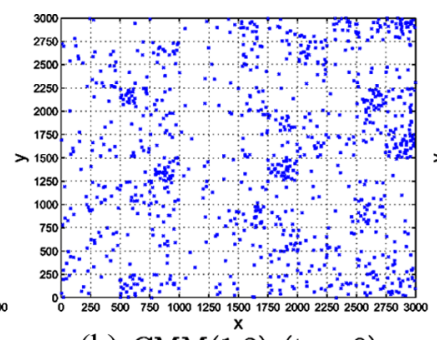

(b) $\operatorname{CMM}(1.2)(\mathrm{t}=0)$

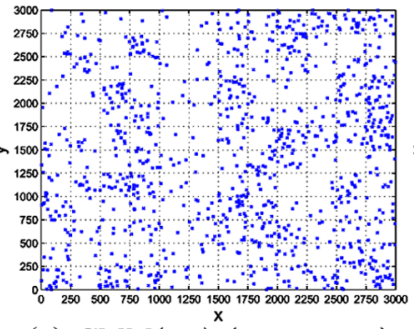

(e) $\operatorname{CMM}(1.2)(\mathrm{t}=10,000)$

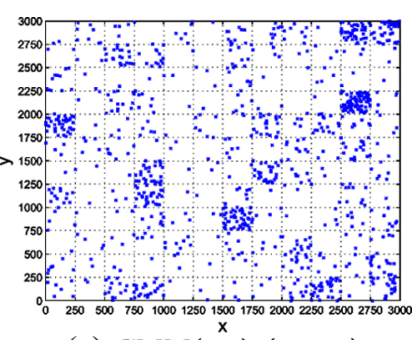

(c) $\operatorname{CMM}(1.4)(\mathrm{t}=0)$

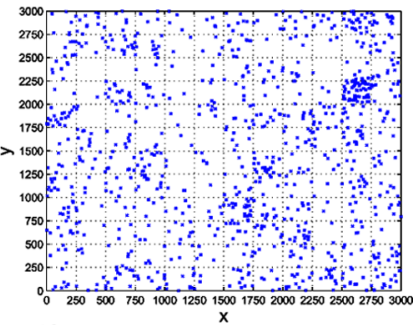

(f) $\operatorname{CMM}(1.4)(\mathrm{t}=10,000)$ 
Definition: In order to analyse the node distribution at steady-state, we will derive $P_{k}$, the probability that a subarea has $k$ nodes in the steady-state. Then, $P_{k}=\sum_{k_{i}} p_{k_{i}} q_{k_{i}, k}$, where $p_{k_{i}}$ denotes the probability that subarea $i$ has $k_{i}$ nodes at the initial stage (after the growth process). $q_{k_{i}, k}$ denotes the conditional probability that the subarea will have $k$ nodes in the steady state assuming $k_{i}$ nodes in the initial stage.

By definition, $p_{k_{i}}=c k_{i}^{-\beta}$ when the growth process ends, where $c$ is a constant. And, $\varphi_{i}$ can be computed using the mobility fraction mentioned earlier. In other words,

$$
\varphi_{i}=\frac{\left(k_{i}+1\right)^{\alpha}}{\Sigma_{j}\left(k_{j}+1\right)^{\alpha}}(1-\xi)+\frac{1}{s_{t}} \xi
$$

The first part is the contribution during the pause time, which depends on the principle of preferential attachment, and the second part is the contribution during the move time, which is assumed to be uniform across all subareas in the network. Since each node is positioned in a subarea $i$ independently with the probability $\varphi_{i}$, it is followed by

$$
q_{k_{i}, k}=\frac{z_{i}^{k} \mathrm{e}^{-z_{i}}}{k !}
$$

where $z_{i}=n \varphi_{i}$ and $n$ is the total number of nodes in the network.

Now, $P_{k}$ can be written as

$$
P_{k}=\sum_{k_{i}} p_{k_{i}} q_{k_{i}, k}=\sum_{k_{i}} c k_{i}^{-\beta} \frac{z_{i}^{k} \mathrm{e}^{-z_{i}}}{k !},
$$

where $z_{i}=n \varphi_{i}$.

To better understand the node dynamics represented by $P_{k}$, let us consider the case $\alpha=1$ for simplicity. In fact, this is called linear preferential attachment (Barabasi and Bonabeau, 2003). Researchers in the scale-free network area have observed that the mechanism of preferential attachment should be linear; if it is faster than linear, the network eventually assumes a star topology with a highly concentrated central hub. However, in CMM, the linear preferential attachment fails to capture the intended level of node clustering unless $\xi$ is zero. This is not surprising because the way we construct the network is not the same as in conventional scale-free networks in the sense that rewiring occurs in the absence of network growth. Then,

$$
\varphi_{i}=\frac{k_{i}+1}{n}(1-\xi)+\frac{1}{s_{t}} \xi
$$

and

$$
z_{i}=\left(k_{i}+1\right)(1-\xi)+\frac{n}{s_{t}} \xi
$$


For a sparse subarea $i$ whose number of nodes is smaller than the average, i.e., $k_{i} \ll \frac{n}{s_{t}}$,

$$
z_{i} \approx \frac{n}{s_{t}} \xi,
$$

and thus

$$
q_{k_{i}, k} \approx \frac{\left(\frac{n}{s_{t}} \xi\right)^{k} \mathrm{e}^{-\frac{n}{s_{t}} \xi}}{k !},
$$

if we assume $\xi \approx 1-\xi$. In other words, a sparse subarea that has a smaller number of nodes than the average, tends to gain more nodes and its average approaches to $\frac{n}{s_{t}}$ with the factor $\xi$.

For a dense subarea $i$ that has a larger number of nodes than the average, i.e., $k_{i} \gg \frac{n}{s_{t}}$,

$$
z_{i} \approx\left(k_{i}+1\right)(1-\xi) \approx k_{i}(1-\xi) .
$$

This yields

$$
q_{k_{i}, k} \approx \frac{\left(k_{i}(1-\xi)\right)^{k} \mathrm{e}^{k_{i}(1-\xi)}}{k !},
$$

which means that the number of nodes after the growth $\left(k_{i}\right)$ decays with the factor $(1-\xi)$.

\section{Performance evaluation}

This section evaluates CMM in comparison to RWP with respect to network capacities. As in the last section, we use 1000 mobile nodes located in a $3000 \times 3000 \mathrm{~m}^{2}$ rectangular area. Each node follows either CMM or RWP with a maximum node speed of $20 \mathrm{~m} / \mathrm{s}$ and a minimum node speed of $5 \mathrm{~m} / \mathrm{s}$. The pause time ( $T_{\text {pause }}$ ) is set to $106 \mathrm{~s}$ to make the mobility factor $50 \%$. The radio transmission range is assumed to be $250 \mathrm{~m}$. Figure 4 shows the node connectivity after the growth step and at steady-state. Nodes in hub subareas have been relocated to achieve the level of node concentration at steady-state. Figure 4(a) corresponds to the statistics just after the growth step and Figure 4(b) corresponds to those at steady-state. They show the cumulative probability of nodes that has the corresponding connectivity.

For RWP, steady-state data was measured after executing the simulation for $10,000 \mathrm{~s}$. As seen in the figures, RWP demonstrates very different connectivity distribution than CMM, which clearly shows a heavy-tail as in Figure 4. This heavy-tail explains the existence of hub subareas. Also, as expected, the heavy-tail of CMM is larger just after the growth step (Figure 4(a)) compared to that at steady state (Figure 4(b)).

Since our primary goal is to understand the maximum achievable capacity rather than the mechanism to achieve it, we make several simplifying assumptions, which will be discussed later in this section. Section 5.1 defines three capacity measures and Section 5.2 presents the comparison of CMM and RWP mobility models using an event-driven simulator, CSIM (Schewetman, 1998). 
Figure 4 Node connectivity in RWP and CMM models $(N=1,000,5 \leq v \leq 20.0(\mathrm{~m} / \mathrm{s})$, and $T_{\text {pause }}=106(\mathrm{~s})$ ): (a) after growth and (b) steady state (see online version for colours)

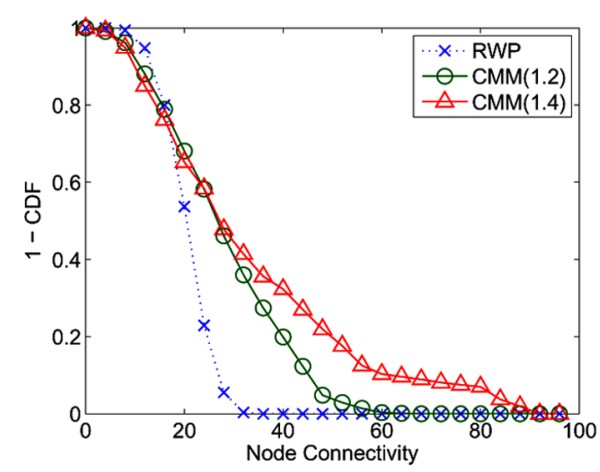

(a)

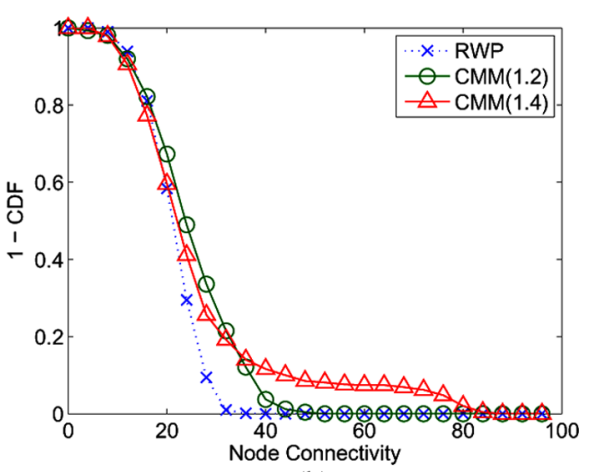

(b)

\subsection{Capacity measures}

Before presenting the network scenario and evaluation results, we define two capacity measures for use in the simulation. They are PHY (nearest one-hop) capacity and MAC (one-hop) capacity.

\subsubsection{PHY (nearest one-hop) capacity}

PHY capacity measures how much traffic the network can support without considering MAC- and network-layer interventions. According to Grossglauser and Tse (2001), the network capacity is constrained by the mutual interference of concurrent transmission between nodes but can be maximised by allocating the channel resource to a node that can best exploit it. This implies communications among the nearest neighbours. Multiple communications can happen simultaneously as long as their Signal-to-Interference Ratio (SIR) is larger than a certain threshold, called capture ratio or $z_{0}$, which is determined by the sensitivity and capability of the radio receiver circuitry (Zorzi and Rao, 1994). Since signal strength greatly depends on the communication distance, a transmission between nearest neighbours can survive with a high probability even in the presence of interference in its proximity.

To elaborate more, for example, node $w$ can withstand the interference (from node $v$ ) and receive a signal from node $u$ correctly as long as SIR is larger than $z_{0}$, i.e.,

$$
\mathrm{SIR}=\frac{P_{t, u} \gamma_{u, w}}{N_{0}+\Sigma_{v \neq u} P_{t, v} \gamma_{v, w}}>z_{0}
$$

where $N_{0}$ is the background noise power, $P_{t, u}$ is node $u$ 's radio transmit power, $\gamma_{u, w}$ is the channel gain from $u$ to $w$ and capture ratio $z_{0}$ ranges from 1 (perfect capture) to $\infty$ (no capture) (Zorzi and Rao, 1994). Under a simple two-ray ground radio propagation model, $\gamma_{u, w} \propto d_{u, w}^{-\theta}$, where $d_{u, w}$ is the $u-w$ distance and the power-loss exponent $\theta$ ranges between nodes 2 and 4 . Assuming that $N_{0}$ is negligible and the 
transmit power is constant, the above equation, for a single interfering node $v$, becomes

$$
\mathrm{SIR}=\frac{P_{t, u} \gamma_{u, w}}{P_{t, v} \gamma_{v, w}}=\frac{\gamma_{u, w}}{\gamma_{v, w}}=\frac{d_{u, w}^{-\theta}}{d_{v, w}^{-\theta}}>z_{0}
$$

where $d_{u, w}$ and $d_{v, w}$ denote the sender-to-receiver $(u-w)$ and interferer-to-receiver $(v-w)$ distance, respectively (Yu et al., 2005).

The PHY capacity can be interpreted as the number of successful concurrent transmissions using a fixed capture ratio $(10 \mathrm{~dB}$ or $6 \mathrm{~dB})$. We expect that the CMM is not poorly performing with respect to PHY capacity because node pairs are determined adaptively depending on the node density, $\rho$.

\subsubsection{MAC (one-hop) capacity}

While nearest-neighbour communication is attractive with respect to PHY capacity, network layer protocols developed for multihop networks usually favour farthest-neighbour communications so as to minimise the hop count to the final destination. In conventional Carrier Sense (CS) based MAC protocols, such as IEEE 802.11 DCF (IEEE, 1999), the PHY capacity cannot be achievable due to carrier sensing. When a node observes a carrier signal above the CS threshold, it holds up pending transmission requests to avoid collisions. In this paper, MAC capacity measures the number of concurrent transmissions, each of which communicates with the farthest neighbour within the transmit range of a sender and offers higher SIR than a fixed capture ratio, e.g., $10 \mathrm{~dB}$. A complete coordination is assumed so that no hidden or exposed terminal (Stallings, 2002) exists.

\subsection{Performance analysis}

This subsection describes simulation results on PHY, MAC and NET capacity with respect to RWP and CMM. We observed that CMM exhibits a lower MAC capacity than RWP as shown in Figure 7, which is due to the excessive congestion in dense subareas and underutilisation in sparse subareas. However, in terms of PHY capacity, CMM performs on par with RWP as in Figures 5 and 6.

\subsubsection{PHY capacity}

Figure 5 compares average PHY capacity of $\mathrm{CMM}(1.2)$ and $\mathrm{CMM}(1.4)$ with respect to RWP. For this experiment, we need to determine the path loss exponent $(\theta)$, which is a key parameter in packet radio communication. Propagation in the mobile channel is described by means of three effects: attenuation due to distance between the sender and the receiver, shadowing due to the lack of visibility between the two nodes, and fading due to multipath propagation. The most popular two-ray ground propagation model is a simple propagation model that considers only the path loss due to communication distance. In other words, the mean received signal power follows an inverse distance power loss law, where an exponent assumes values between 2 and 4 , and is typically 4 in land mobile radio environments. In the $915 \mathrm{MHz}$ WaveLAN radio hardware, the transmit power is $24.5 \mathrm{dBm}$ and the receive 
sensitivity is $-72 \mathrm{dBm}$, which is translated to $250 \mathrm{~m}$ or shorter distance between the sender and the receiver for successful communication.

Figure 5 PHY capacities in RWP and CMM models $(N=1,000,5 \leq v \leq 20.0(\mathrm{~m} / \mathrm{s})$,

$T_{\text {pause }}=106$ (s)): (a) $\theta=2, z_{0}=10 \mathrm{~dB}$; (b) $\theta=3, z_{0}=10 \mathrm{~dB}$; (c) $\theta=2$,

$z_{0}=6 \mathrm{~dB}$ and (d) $\theta=3, z_{0}=6 \mathrm{~dB}$ (see online version for colours)
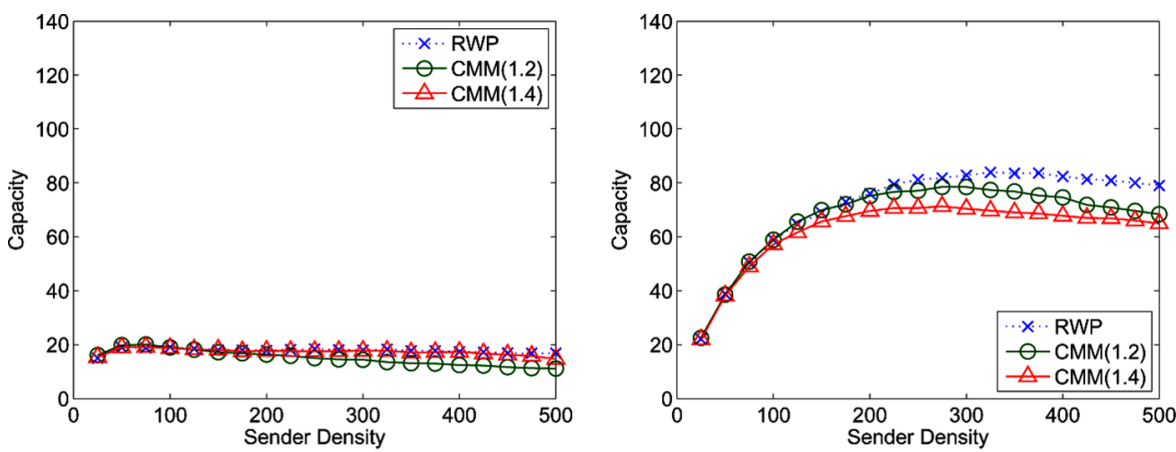

(a)

(b)

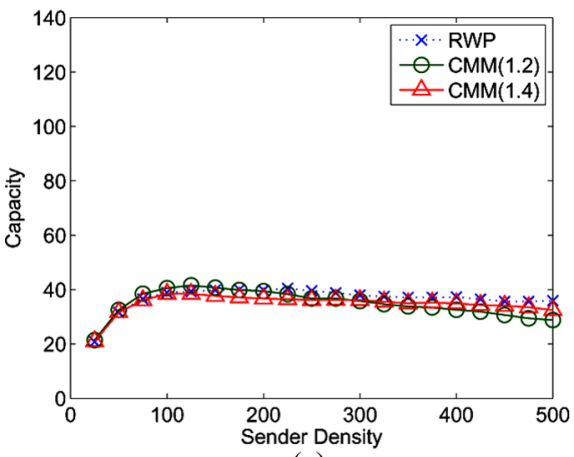

(c)

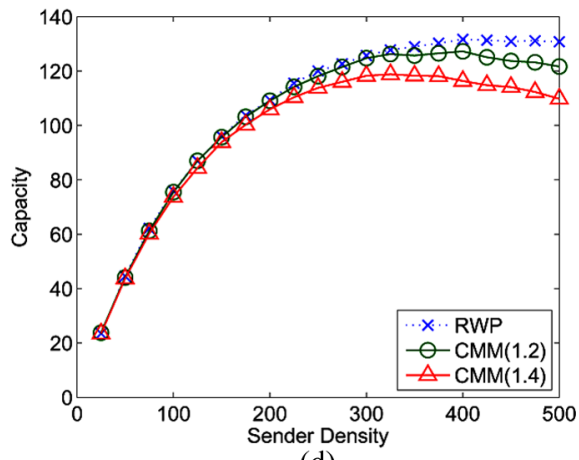

(d)

Figure 6 PHY link capacities in RWP and CMM models $(N=1,000,5 \leq v \leq 20.0(\mathrm{~m} / \mathrm{s})$, $T_{\text {pause }}=106(\mathrm{~s}), z_{0}=10 \mathrm{~dB}$ ): (a) $\theta=2$ and (b) $\theta=3$ (see online version for colours)

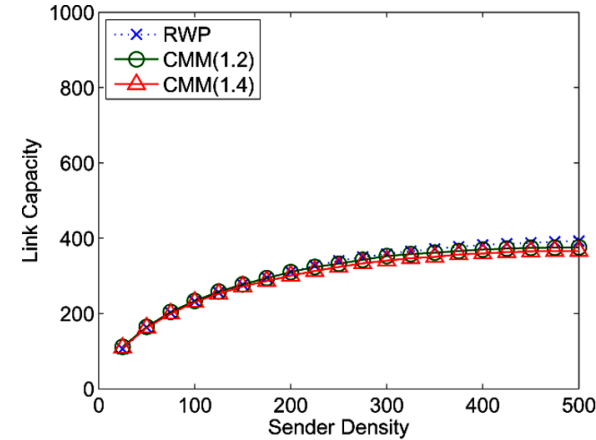

(a)

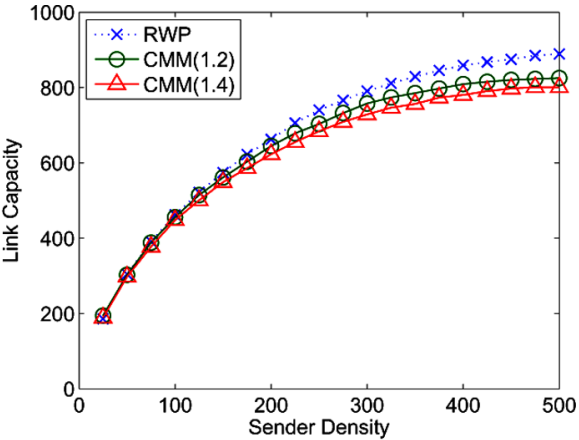

(b) 
Figure 7 MAC capacities in RWP and CMM models $(N=1,000,5 \leq v \leq 20.0(\mathrm{~m} / \mathrm{s})$, $T_{\text {pause }}=106(\mathrm{~s}), z_{0}=10 \mathrm{~dB}$ ): (a) $\theta=2$ and (b) $\theta=3$ (see online version for colours)

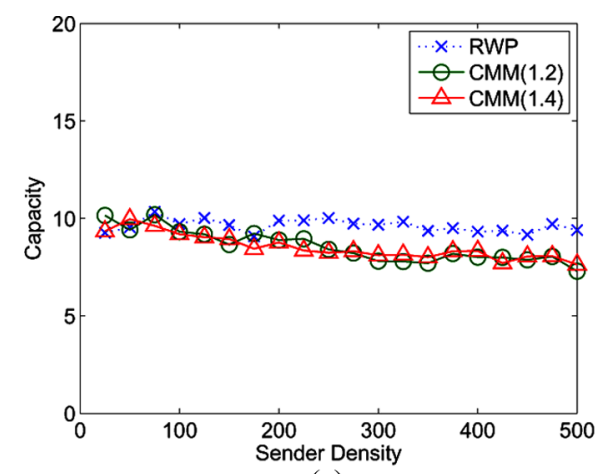

(a)

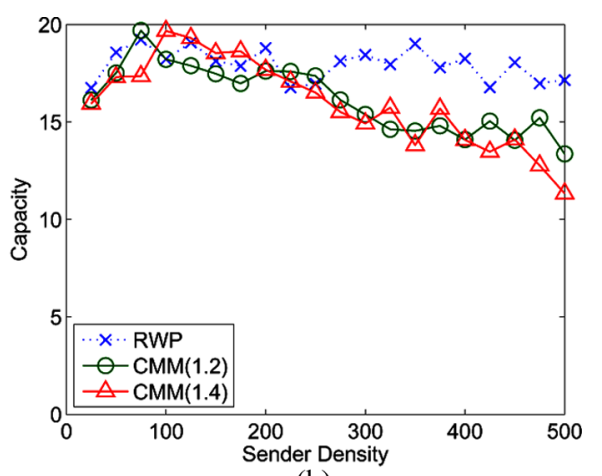

(b)

As shown in the Figure 5, CMM exhibits comparable performance with respect to RWP. With a larger path loss exponent, signal strength attenuates more rapidly and therefore, it opens a window for other pairs to communicate increasing the communication concurrency. Figure 5(c) and (d) show a similar comparison with the capture ratio of $6 \mathrm{~dB}$, which means the communication has a better chance to be successful even if its SIR is smaller. With a smaller capture ratio, the network can achieve a higher throughput but at the cost of increased cost for radio hardware. Capture ratio of $0 \mathrm{~dB}$ represents the case of perfect capture.

Figure 6 shows another PHY capacity, where we do not incorporate the capture ratio. Instead, we assume that each radio device can communicate at any achievable data rate allowed by the radio environment in the proximity. For each pair of nodes, a receiver node calculates its SIR, and thus, the maximum data rate using the Shannon's theorem. Figure 6 shows the aggregate throughput assuming that the link bandwidth is unity. As seen in the figure, CMM performs almost similar to that of RWP. Again, the path loss exponent significantly affects the performance as seen in Figure 6(a) and (b).

\subsubsection{MAC capacity}

Next, we present the results on MAC capacity in Figure 7. As discussed earlier, each of the senders transmits to its farthest possible neighbour within its transmit range. Here, it is shown that RWP performs better than CMM as explained earlier. Simplicity in carrier sense-based MAC protocols comes at the cost of performance loss. In particular, node clustering in CMM negatively affects the network performance. It is also observed that as the sender density increases, the MAC capacity of RWP and CMM decreases due to higher congestion. Path loss exponent plays a key role in determining the maximum performance as well. Comparing Figure 7(a) and (b), we observe that the network throughput is almost double in both of CMM and RWP. 


\section{Discussions, managerial implications and concluding remarks}

The field of wireless networking has received unprecedented attention during the last decade due to its great success as well as even greater potential to create new businesses and opportunities beyond what is being offered by using internet. Wireless LANs, known as Wi-Fi hot spots, have become prevalent in modern enterprises as well as public and residential areas. Numerous efforts, planned or unplanned, have been made to provide internet connectivity over a larger geographical area based on wireless mesh (Rooftop@Media; NYCwireless; MetroFi; SeattleWireless) and WiMAX technologies. Seamless convergence with $3 \mathrm{G}$ and $4 \mathrm{G}$ cellular networks would offer an unlimited set of interesting applications.

However, the strategic positioning of wireless networks in the value chain requires an enumeration of a large number of factors, namely risk assessment of potential investment under mobility, characterisation of QoS, knowledge transfer of management and control experiences across an extended enterprise (Shriram et al., 2008; Kivi, 2009). Historically, the recognition of value-centred strategic understanding of mobile IT has been considered the key source of organisational competitive advantages (Sheng et al., 2005). This approach identifies the major strategic implications of mobile IT in improvement of working process, increment of internal knowledge management and enhancement of sales and marketing effectiveness.

Among those risk factors, mobility is the most fundamental and challenging risk factor in wireless networks. For example, when a mobile user talks on the phone using a Voice over IP (VoIP) application, a legitimate question is whether the end-to-end delay can be bounded, say not greater than $50 \mathrm{~ms}$ (ITU-T recommendation G.114, 1993), in the presence of mobility. If this cannot be met in a large faction of cases, the corresponding technology is not recommended for adoption. Therefore, it is not an overstatement that the study on mobility has important managerial implications from the mobile IT point of view.

The main theme of this paper is to develop a new mobility model, called Clustered Mobility Model (CMM), which allows non-homogeneous node distribution driven by two principles: network growth and preferential attachment. The level of non-homogeneity is controllable in CMM by changing the clustering exponent, $\alpha$, and is engineered not to vary during the simulation. For this, we represent the non-homogeneity with the distribution of subarea population and mobility fraction, $\xi$. The main contribution of this paper is to consider the rationale behind the move and make it a driving force in the mobility model. We believe, if properly stipulated and employed, the behaviour-based mobility model would better represent the real-life scenarios.

To assess the strength and weakness of the scale-free phenomena, this paper defines network capacities at PHY and MAC layers. Based on simulation-based performance analysis, we observed that the network with CMM exhibits lower MAC capacity but achieve as high PHY capacity as conventional mobility model such as RWP. This suggests us that the network with CMM requires unique network protocols to optimise performance. We strongly believe that the proposed CMM can be usefully used to investigate the properties of networks that are likely to occur in a real deployment of wireless multihop networks. 
For the future work, we plan to evaluate the network or multihop capacity in addition to PHY (nearest one-hop) and MAC (one-hop) capacity. Since the ultimate goal of a wireless ad hoc network is to deliver packets to the desired destination, it is important to measure the robustness of multihop connections between a pair of nodes in the network. We also plan to delve into routing/multicasting issues under the CMM in multihop networks. Due to the presence of hub nodes, it is essential to reduce network congestion/collision that may cause serious network performance degradation.

\section{Acknowledgements}

This research was supported in part by US National Science Foundation grants CNS-0831673, CNS-0831853, and CNS-0821319, the Startup grant in the Department of Computer Science at Texas Tech University, and the World Class University (WCU) programme through the Korea Science and Engineering Foundation funded by the Ministry of Education, Science, and Technology (Project No. R31-2008-000-10100-0).

\section{References}

Albert, R. and Barabasi, A.L. (2000) 'Topology of evolving networks: local events and universality', Physical Review Letters, Vol. 85, No. 24, pp.5234-5237.

Alchaita, M. (2008) 'Link longevity and mobility in self-dependent multi-hop mobile environments', International Journal of Mobile Communications, Vol. 6, No. 4, pp.519-539.

Bar-Noy, A., Kessler, I. and Sidi, M. (1995) 'Mobile users: to update or not to update?', Wireless Networks, Vol. 1, No. 2, pp.175-185.

Barabasi, A.L. and Bonabeau, E. (2003) 'Scale-free networks', Scientific American, Vol. 288, pp.50-59.

Bettstetter, C., Resta, G. and Santi, P. (2003) 'The node distribution of the random waypoint mobility model for wireless ad hoc newtorks', IEEE Trans. on Mobile Computing, Vol. 2, No. 3, pp.257-269.

Bettstetter, C., Hartenstein, H. and Perez-Costa, X. (2004) 'Stochastic properties of the random waypoint mobility model', Wireless Networks, Vol. 10, No. 5, pp.555-567.

Blough, D.M., Resta, G. and Santi, P. (2004). 'A statistical analysis of the long-run node spatial distribution in mobile ad hoc networks', Wireless Networks, Vol. 10, No. 5, pp. $543-554$

Camp, T., Boleng, J. and Davies, V. (2002) 'A survey of mobility models for ad hoc network research', Wireless Communications Mobile Computing (WCMC): Special Issues on Mobile Ad Hoc Networking: Research, Trends, and Applications, Vol. 2, No. 5, pp.483-502.

Cano, J-C., Manzoni, P. and Sanchez, M. (2004) 'Evaluating the impact of group mobility on the performance of mobile ad hoc networks', Proc. IEEE ICC, pp.4039-4043.

Centonza, A., Taylor, G.A., Itagaki, T., Owens, T.J., Cosmas, J. and Song, Y. (2006) 'Stochastic user behaviour modelling and network simulation for resource management in cooperation with mobile telecommunications and broadcast networks', International Journal of Mobile Communications, Vol. 4, No. 1, pp.97-117. 
Chen, Q., Chang, H., Govindan, R., Jamin, S., Shenker, S. and Willinger, W. (2002) 'The origin of power laws in internet topologies revisited', IEEE INFOCOM, New York, NY, USA, pp.608-617.

Erdös, P. and Rényi, A. (1960) 'On the evolution of random graphs', Publ. Math. Inst. Hungar. Acad. Sci., Vol. 5, pp.17-61.

Grossglauser, M. and Tse, D. (2001) 'Mobility increases the capacity of ad-hoc wireless networks', IEEE INFOCOM, Anchorage, AK, USA, pp.1360-1369.

Herrmann, C., Barthélemy, M. and Provero, P. (2003) Connectivity Distribution of Spatial Networks, e-print cond-mat/0302544, February.

Hsu, W., Spyropoulosy, T., Psounisz, K. and Helmy, A. (2007) 'Modeling time-variant user mobility in wireless mobile networks', Proc. IEEE INFOCOM, Anchorage, AK, USA, pp.758-766.

IEEE. IEEE Std 802.11-1999 (1999) Local and Metropolitan Area Network, Specific Requirements, Part 11: Wireless LAN Medium Access Control (MAC) and Physical Layer (PHY) Specifications, http://standards.iee.org/getieee802/download/802-111999.pdf

Jardosh, A., Belding-Royer, E.M., Almeroth, K. and Suri, S. (2003) 'Towards realistic mobility models for mobile ad hoc networks', Proc. IEEE MOBICOM, San Diego, CA, USA, pp.217-229.

Johnson, D.B. and Maltz, D.A. (1996) 'Dynamic source routing in ad hoc wireless networks', Mobile Computing, Kluwer, pp.153-181.

Jolly, V. and Latifi, S. (2007) 'Reliable data transmission in mobile ad hoc sensor networks', International Journal of Mobile Communications, Vol. 5, No. 5, pp.558-571.

Kawadia, V. and Kumar, P.R. (2003) 'Power control and clustering in ad hoc networks', Proc. IEEE INFOCOM, San Francisco, CA, USA, pp.459-469.

Kivi, A. (2009) 'Measuring mobile service usage: methods and measurement points', International Journal of Mobile Communications, Vol. 7, No. 4, pp.415-435.

Medina, A., Matta, I. and Byers, J. (2000) 'On the origin of power laws in internet topologies', ACM SIGCOMM Computer Communication Review, Vol. 30, No. 2, pp.18-28.

Németh, G. and Vatty, G. (2003) Giant Clusters in Random Ad Hoc Networks, e-print cond-mat/0211325.

Oppenheim, N. (1995) Urban Travel Demand Modeling: From Individual Choices to General Equilibrium, Wiley-Interscience.

Rhee, I., Shin, M., Hong, S., Lee, K. and Chong, S. (2007) 'Human mobility patterns and their impact on routing in human-driven mobile networks', Proc. ACM HotNets-VI, Atlanta, GA, USA.

Schewetman, H. (1998) CSIM User's Guide, (Version 18), MCC Corp. http://www. mesquite.com

Sheng, H., Fui-Hoon, F. and Siau, K. (2005) 'Strategic implications of mobile technology: a case study using value-focused thinking', Journal of Strategic Information Systems, Vol. 4, pp.269-290.

Shriram, R., Sugumaran, V. and Vivekanandan, K. (2008) 'A middleware for information processing in mobile computing platforms', International Journal of Mobile Communications, Vol. 6, No. 5, pp.646-666.

Stallings, W. (2002) IEEE 802.11 Wireless LAN Standard, Prentice-Hall, Inc., Chapter 14, Wireless Communications and Networks.

Strogatz, S.H. (2001) 'Exploring complex networks', Nature, Vol. 410, pp.268-276. 
Sullivan, R. (2001) One Nation: America Remembers September 11, 2001, Time Warner Trade Publishing.

Wang, K. and Li, B. (2002a) 'Group mobility and partition prediction in wireless ad-hoc networks', Proc. IEEE ICC, Anchorage AK, USA, pp.1017-1021.

Wang, K.H. and Li, B. (2002b) 'Efficient and guaranteed service coverage in partitionable mobile ad-hoc networks', Proc. IEEE INFOCOM, New York, NY, USA, pp.1089-1098.

Watts, D.J. and Strogatz, S.H. (1998) 'Collective dynamics of 'small-world' networks', Nature, Vol. 393, pp.440-442.

Yoon, J., Liu, M. and Noble, B. (2003) 'Random waypoint considered harmful', Proc. IEEE INFOCOM., San Francisco, CA, USA, pp.1312-1321.

Yu, C., Shin, K.G. and Song, L. (2005) 'Link-layer salvaging for making routing progress in mobile ad hoc networks', Proc. MobiHoc, Urbana-Champaign, IL, USA, pp.242-254.

Zhou, B., Xu, K. and Gerla, M. (2004) 'Group and swarm mobility models for ad hoc network scenarios using virtual tracks', Proc. IEEE Milcom, Monterey, CA, USA, pp.289-294.

Zorzi, M. and Rao, R. (1994) 'Capture and retransmission control in mobile radio', IEEE Journal on Selected Areas in Communications, Vol. 12, No. 8, pp.1289-1298.

\section{Websites}

ITU-T Recommendation G.114, International Telecommunication Union, Tech. Rep.

MetroFi Portland Free Wi-Fi, [Online], http://www.metrofiportland.com

NYCwireless, [Online], http://NYCwireless.net

Rooftop@Media,[Online], http://rooftops.media.mit.edu

SeattleWireless, [Online], http://SeattleWireless.net 